\title{
PLHER (radio plaquetas/hematocrito): una herramienta para discriminar pacientes adultos atendidos en la sala de emergencias con o sin sangrado agudo.
}

\author{
Aníbal A. Teherán ${ }^{1 *}$ Andrés Ochoa ${ }^{2}$, Luis M. Pombo ${ }^{3}$, Daniel M. Núñez ${ }^{4}$, Oscar L. Gómez ${ }^{4}$, Albert A. Ávila ${ }^{5}$ \\ Luis G. Guerrero ${ }^{6}$, Norma Montoya ${ }^{7}$.
}

\author{
'Médico Epidemiólogo, Grupo de Investigación COMPLEXUS, Fundación Universitaria Juan N. Corpas. Bogotá, Colombia \\ ${ }^{2}$ Médico General, Sala de Emergencias, Clínica del Occidente S.A. Bogotá, Colombia \\ ${ }^{3}$ Ingeniero Químico MSc, Grupo de Investigación GIFVTA, Fundación Universitaria Juan N. Corpas. Bogotá, Colombia \\ ${ }^{4}$ Residentes de Medicina de Emergencias, Colegio Mayor Nuestra Señora del Rosario. Bogotá, Colombia \\ ${ }^{5}$ Especialista en Medicina de Emergencias, Grupo de Investigación ANTHUS, Los Cobos Medical Center. Estudiante de Maestría en Salud Pública, Universidad \\ de la Sabana. Bogotá, Colombia \\ ${ }^{6}$ Médico Gastroenterólogo, Clínica del Occidente S.A.. Bogotá, Colombia \\ ${ }^{7}$ Bacterióloga, Directora de Laboratorio Clínico, Clínica del Occidente S.A, Bogotá, Colombia
}

\section{RESUMEN}

Objetivo: La aproximación al diagnóstico de sangrado agudo (SA) manifestado con síntomas inespecíficos, utilizando el hematocrito como laboratorio de apoyo es un reto en la Sala de Emergencias (SE). Se compararon las capacidades operativas del hematocrito y radio plaqueta/hematocrito (PLHER) para discriminar pacientes con SA.

Métodos: Con un diseño de precisión diagnóstica, se compararon pacientes con y sin SA atendidos en la SE de la Clínica del Occidente S.A. durante el 2017. El espectro de enfermedad (EDx) incluyó controles negativos[grupo_0], población general con o sin trauma ortopédico cerrado[grupos_1-2], pacientes con trauma ortopédico cerrado [grupo_3], con SA ginecológico [grupo_4], SA gastrointestinal [grupo_5] y controles positivos [grupos_6-7]. Se compararon las capacidades operativas del PLHER y hematocrito para discriminar pacientes con SA.

Resultados: 655 pacientes fueron seleccionados, principalmente hombres. No hubo diferencias entre las medianas de hematocrito del grupo_0 con los grupos 1-2; comparado con el grupo 0, la mediana del PLHER fue mayor en todos los grupos del EDx. (p: $<0.05)$. Con los puntos de corte $\geq 6.037 \mathrm{y} \geq 6.348$ el AUC del PLHER para discriminar pacientes con posible SA [grupos 1-3] o cualquier grupo del EDx [grupos 1-7] fue 0.709 y 0.766 , respectivamente. Los AUC del hematocrito para discriminar pacientes con posible SA o cualquier grupo del EDx fueron 0.682 y 0.755 , respectivamente.

Discusión: El PLHER presentó mejores capacidades operativas que el hematocrito para discriminar pacientes con SA. Podría ser útil para identificar pacientes con SA atendidos con signos y síntomas inespecíficos en la SE.

Palabras clave: Sangrado agudo, Hematocrito, PLHER, Hemorragia, Precisión diagnóstica, Emergencias.

\section{ABSTRACT}

"PLHER" (Platelet / Hematocrit Ratio): A tool to discriminate adult patients assisted at emergency room with or without acute bleeding.

Aim: The diagnosis of acute bleeding (AB), manifested with nonspecific, using hematocrit, as a support laboratory is a challenge in the Emergency Room (ER). Ratio platelet / hematocrit (PLHER) operational capabilities to discriminate patients with $\mathrm{AB}$ were compared.

Methods: With a diagnostic accuracy design, patients with and without $\mathrm{AB}$ treated in the ER of Clínica del Occidente S.A. were compared, during 2017. The disease spectrum (EDx) included negative controls [group_0], general public with or without closed orthopedic injuries [groups_1-2], patients with closed orthopedic injuries [group_3], with gynecological $\mathrm{AB}$ [group_4], gastrointestinal [group_5] and positive controls [groups_6-7]. It was compared the operational capabilities of PLHER and hematocrit to discriminate patients with $\mathrm{AB}$.

Results: 655 patients were selected, mainly men. None differences were observed between hematocrit medians of group_0 and groups_12. Compared to group_0, the median PLHER was higher in all EDx groups ( $\mathrm{p}:<0.05)$. The AUC of the PLHER, to discriminate patients with possible $\mathrm{AB}$ [groups 1-3] or any group of the EDx were 0.709 and 0.766 , respectively, considering the cut-off points $\geq 6.037$ and $\geq 6.348$. The AUC of the hematocrit to discriminate patients with possible $\mathrm{AB}$ or any group of EDx were 0.682 and 0.755 , respectively.

Discussion: The PLHER showed better operational capabilities than hematocrit to discriminate patients with $\mathrm{AB}$. It could be a useful tool to identify patients with $\mathrm{AB}$ treated with non-specific signs and symptoms in ER

Keywords: Acute bleeding, Hematocrit, PLHER, Hemorrhage, Diagnostic accuracy, Emergencies.

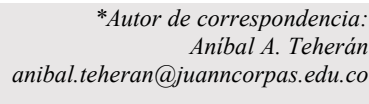

Como citar: Teherán, AA*, Ochoa, A, Pombo, LM, Núñez DM, Gómez, OL, Avila, AA, Guerrero, LG, Montoya, M. PLHER (radio plaquetas/hematocrito): una herramienta para discriminar pacientes adultos atendidos en la sala de emergencias con o sin sangrado agudo. Revista Cuarzo 2019;25(2):21-27

Recibido: 12 de julio de 2019 Aceptado: 20 de septiembre de 2019 Publicado: 30 de diciembre de 2019

DOI: https://doi.org/10.26752/cuarzo.v25.n2.443

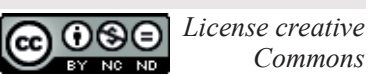




\section{INTRODUCCIÓN}

$\mathrm{E}$ 1 SA en adultos atendidos en la SE podría originarse en causas médicas (SAM) o traumáticas (SAT). En hombres y mujeres no embarazadas las causas médicas más frecuentes de SA gastrointestinal (SGI), genitourinario, hematológico e inducido por medicamentos. La incidencia es variable según la causa de SAM, desde 50-150/100.000 atenciones anuales por SGI alto, hasta $2-5 \%$ de sangrados mayores en usuarios de anticoagulantes orales (4).

Las áreas anatómicas de donde proviene el SAM frecuentemente expresan signos y síntomas específicos como hematemesis, melenas, dolor pélvico, sangrado vaginal, hemoptisis o coloración de la piel (1-4). Adicionalmente, en el SAT la aproximación al sangrado mayor debe incluir el mecanismo y cinemática del trauma (5).

Aun con una detallada historia clínica, signos y síntomas como la palidez, taquicardia e hipotensión aislada son inespecíficos e imprecisos para discriminar entre SA activos ocultos y presentaciones agudas de enfermedades autoinmunes, infecciosas o hematológicas $(5,6)$.

En la aproximación al SGI, por ejemplo, el nitrógeno ureico, creatinina, radio $\mathrm{BUN} /$ creatinina, melena y los puntajes de escalas son buenos predictores clínicos, pero moderadamente útiles para discriminar pacientes con SA sin "alta probabilidad a priori”, además, las escalas están limitadas por la cantidad de parámetros que las conforman y el tiempo necesario para medirlos (7-9).

Las decisiones clínicas sobre la causa y tratamiento temprano del SA frecuentemente se apoyan en la interpretación del hemograma y la concentración de hemoglobina o hematocrito, pero su utilidad es limitada por la probabilidad de falsos negativos en el diagnóstico de anemia aguda dada la hemoconcentración secundaria a la pérdida de volumen en el SA (10).

El hemograma es un recurso de fácil acceso, económico, que aporta resultados preliminares en $<30$ segundos, además reporta diferentes líneas celulares que participan en la hemostasia durante el SA, y al igual que lo demostrado en urgencias oncológicas o procesos inflamatorios agudos, la interpretación relativa de las líneas celulares del hemograma podría reflejar con mayor precisión procesos dinámicos que ocurren durante el SA $(11,12)$.

Por las relaciones hemostásicas compartidas entre las plaquetas y eritrocitos durante el SA, el objetivo de esta investigación fue determinar si el PLHER podría discriminar con mayor precisión que el hematocrito a pacientes con SA, principalmente en la población general, con sangrados no aparentes o en fases tempranas de choque hemorrágico.

\section{MATERIALES Y MÉTODOS}

Metodología: Diseño, lugar y selección de pacientes.

Con un diseño de precisión diagnóstica, se utilizaron los registros de historia clínica y resultados de hemogramas de pacientes atendidos en la CDO durante el año 2017 (muestra por conveniencia).

Se incluyeron ocho subgrupos de pacientes mayores de 18 años para cubrir el EDx. Se excluyeron pacientes remitidos de otra institución, con recuento de plaquetas menor a 150.000 o mayor a 750.000, sin hemograma solicitado durante la atención inicial en el SE, con historia de anemia crónica, y en el subgrupo de pacientes no transfundidos, pacientes con hematocrito menor de 21 o con choque mayor a grado II al ingreso. Esta investigación fue aprobada por el Comité de Investigación de la CDO.

\section{Espectro diagnóstico.}

Los pacientes fueron independientes entre subgrupos, es decir, los pacientes incluidos en un subgrupo específico no pertenecían al mismo tiempo a otro subgrupo, excepto en el subgrupo de transfundidos, en quienes con los resultados de hemogramas pre y postransfusión se crearon dos subgrupos. Los pacientes de cada subgrupo se identificaron con listados de atenciones realizadas en cada departamento o área de atención. Grupo 0 - controles negativos: pacientes sin SA, no consumidores de antiplaquetarios o anticoagulantes, atendidos por una presuntiva enfermedad cardíaca, sin comorbilidades, hepáticas, renales, ni hematológicas. Grupo 1 - posible sangrado (a): pacientes pertenecientes a la población general atendida en la SE, que incluye pacientes de cualquier subgrupo del EDx, excepto con trauma ortopédico o pre-postransfusión. del espectro excepto pacientes transfundidos o atendidos por fracturas de huesos largos. Grupo 2 - posible sangrado (b): pacientes de la población general atendida en la sala de emergencias, que incluye cualquiera de los subgrupos del espectro (con o sin SA) excepto los pacientes transfundidos. Grupo 3 - posible sangrado (c): pacientes atendidos por fracturas cerradas o abiertas en pelvis o de huesos largos, tanto en miembros superiores, inferiores, o ambos. Grupo 4 sangrado evidente/confirmado (GO): pacientes atendidas en el servicio de ginecología y obstetricia con hemorragia uterina o hemorragia en el primer trimestre. Grupo 5 sangrado evidente/confirmado (GI): pacientes atendidos por síntomas sugestivos de SGI, con diagnóstico endoscópico de ulcera Forrest IIa o Forrest IIb. Grupo 6 - control positivo (posttransf): pacientes del grupo 6 en estado de postransfusión de glóbulos rojos empaquetados secundario a SA por etiologías de traumáticas, gastrointestinales o ginecobstétricas. Grupo 7 control positivo (pre-transf): pacientes con indicación de transfusión de glóbulos rojos empaquetados secundaria a SA por etiologías de traumáticas, gastrointestinales $\mathrm{O}$ ginecobstétricas 


\section{Recolección de datos y variables.}

Se solicitaron listados de los subgrupos pertenecientes al EDx y con la información contenida en los registros clínicos clínicos, resultados del primer hemograma en el SE o hemogramas pre y postransfusión entregados por el Laboratorio Clínico del CDO S.A., se recolectaron las variables sexo, edad, causa específica de sangrado, conteo absoluto de plaquetas, valor de hematocrito, indicación de transfusión de glóbulos rojos empaquetados. La información se almacenó en planillas de Excel ${ }^{\circledR}$.

\section{Variable de resultado.}

Capacidades operativas del hematocrito y PLHER para discriminar pacientes con SA atendidos en el SE.

\section{Análisis estadístico.}

El radio plaqueta hematocrito (PLHER) se construyó al dividir el conteo de plaquetas entre 1000 y posteriormente al dividir las plaquetas sobre el hematocrito:

- PLHER: [Conteo de plaquetas/1000] / hematocrito

Los datos se expresaron en proporciones y medianas. Se rechazó normalidad con la prueba Kolmogorov Smirnov (KS). Se estableció una $\mathrm{p}:<0.05$ a una cola como significativa. Los pacientes de los subgrupos 1-7 se clasificaron como 1 , y los controles negativos como 0 ; posteriormente se calcularon las capacidades operativas del PLHER y hematocrito para discriminar entre controles negativos y pacientes con posible SA (grupos 1-3; SA no evidente) o pacientes del EDx (grupos 1-7). Se utilizaron los paquetes estadísticos SPSS 24, Minitab 18 y XLSTAT para analizar los datos y diseñar las figuras.

\section{RESULTADOS}

\section{Características generales.}

Se recolectaron datos de 792 pacientes atendidos consecutivamente en la SE, se excluyeron 137 por información incompleta o incumplimiento con los criterios de elegibilidad. Se rechazó normalidad en la distribución del hematocrito, plaquetas y el PLHER (KS, valor p: $>0.1$ ).

En la tabla 1 y tabla $1 \mathrm{~s}$ (material suplementario) se presentan las características generales de los 655 pacientes seleccionados. La mayoría fueron hombres, el grupo 3 integrado por pacientes con fracturas de huesos largos fue el más representativo (39.5\%) y el grupo 1 con pacientes de la población general sin fracturas fue el menos representativo (4.4\%).

Las medianas (p25-p75; mínimo, máximo) de hematocrito, plaquetas y PLHER, respectivamente fueron 37.9 (30.5-43.0; 21.3, 56.9), 254 (210-318; 150, 723), 6.854 (5.583-9.003; $3.026,29.153)$.
Tabla 1: Características generales

\begin{tabular}{clccccc}
\hline \hline \multirow{2}{*}{ Espectro } & \multirow{2}{*}{$\mathbf{N}$} & Hombres & Mujeres & HE & PL $^{\mathbf{a}}$ & PLHER \\
\cline { 3 - 7 } & & $\mathrm{n}(\%)$ & $\mathrm{n}(\%)$ & \multicolumn{3}{c}{ Medianas } \\
\hline \hline Grupo 0 & 50 & $41(11.3)$ & $9(3.1)$ & 43.8 & 224 & 5.089 \\
Grupo 1 & 29 & $15(4.1)$ & $14(4.8)$ & 44.3 & 256 & 6.031 \\
Grupo 2 & 64 & $22(6.0)$ & $42(14.4)$ & 43.1 & 276 & 6.349 \\
Grupo 3 & 259 & $154(42.3)$ & 105 & 38.1 & 245 & 6.525 \\
Grupo 4 & 46 & $0(0.0)$ & $46(15.8)$ & 38.9 & 299 & 7.648 \\
Grupo 5 & 117 & $82(22.5)$ & $35(12.0)$ & 30.2 & 242 & 8.199 \\
Grupo 6 & 48 & $26(7.1)$ & $22(7.5)$ & 29.5 & 307 & 9.315 \\
Grupo 7 & 42 & 24 (6.6) & $18(6.4)$ & 25.8 & 331 & 11.603 \\
\hline \hline El conteo total de plaquetasa se dividió entre 1.000 para mejorar la interpretación n del PLHER. HE: \\
hematocrito, PL: plaquetas. En la tabla s1 del material suplementario se presentan otros parámetros \\
\multicolumn{3}{c}{ medidos. El grupo 0, sin sangrado, es el referente de comparación. }
\end{tabular}

\section{Características demográficas y parámetros del SA.}

La mediana del conteo de plaquetas y de PLHER fue mayor en mujeres que en hombres (valor $\mathrm{p}:<0.001$ ), pero no se identificaron estas diferencias al comparar la mediana de hematocrito por sexo (Figura 1).

Figura 1: Mediana de PLHER, hematocrito y plaquetas por sexo.

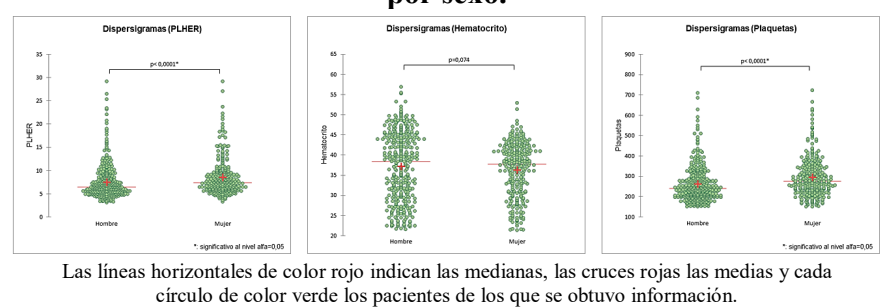

La edad presentó correlación directa con el hematocrito, indirecta con el conteo de plaquetas y ninguna con el PLHER. Este último presentó correlación fuerte y directa con el conteo de plaquetas y moderada e indirecta con el hematocrito (Figura 2).

Por último, se identificó que la correlación entre el PLHER y las plaquetas fue mayor en mujeres (r: 0.837) que en hombres (0.700) (Figura 2).

Figura 2: Correlación entre edad, plaquetas, hematocrito y PLHER.

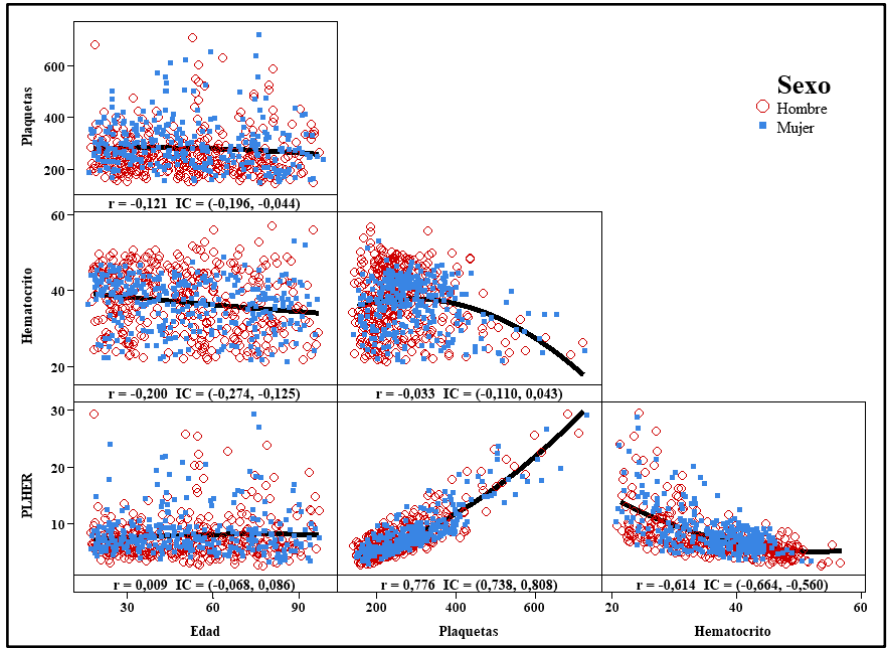

r: correlación de Spearman. La línea de tendencia incluyó el intercepto y se ajustó a una función cuadrática. 


\section{Diferencia de medianas de hematocrito y PLHER} entre grupos del espectro diagnóstico.

Comparado con el grupo 0 (control negativo, referente), en los grupos 1 a 7 se esperaba una mediana de hematocrito más baja y una mediana de PLHER más alta.

Las diferencias de medianas de hematocrito entre el grupo $0 \mathrm{y}$ los grupos del EDx se presentaron desde el grupo 3 en adelante (U Mann Whitney a una cola $\left[\mathrm{H}_{1}: \eta_{1}-\eta_{2}<0\right]$; valor $\mathrm{p}:<0.000$ ), pero al comparar con la mediana de PLHER del grupo 0, las medianas de PLHER de todos los grupos del EDx fueron mayores (U Mann Whitney a una cola $\left[H_{1}: \eta_{1}-\eta_{2}>0\right]$ ] valor $p$ : $<0.05$ ) (Figura 3).

Figura 3: Comparación de medianas de hematocrito y PLHER entre grupos del espectro diagnóstico.

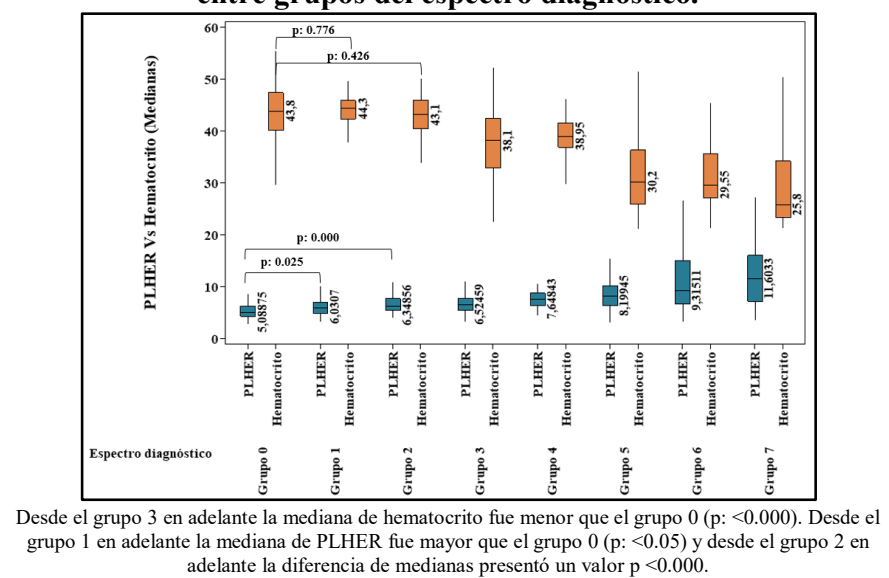

\section{Capacidades operativas de PLHER y hematocrito.}

El PLHER demostró capacidades operativas superiores que el hematocrito para discriminar entre pacientes con SA y pacientes con posible de SA atendidos en la SE grupos 1-3; SA no evidente. Asimismo, el desempeño del PLHER para discriminar pacientes con SA fue más elevado que el hematocrito cuando se incluyeron todos los subgrupos del EDx (Figura 4).

Figura 4: Comparación de AUC entre el PLHER y hematocrito para discriminar pacientes con sangrado agudo.

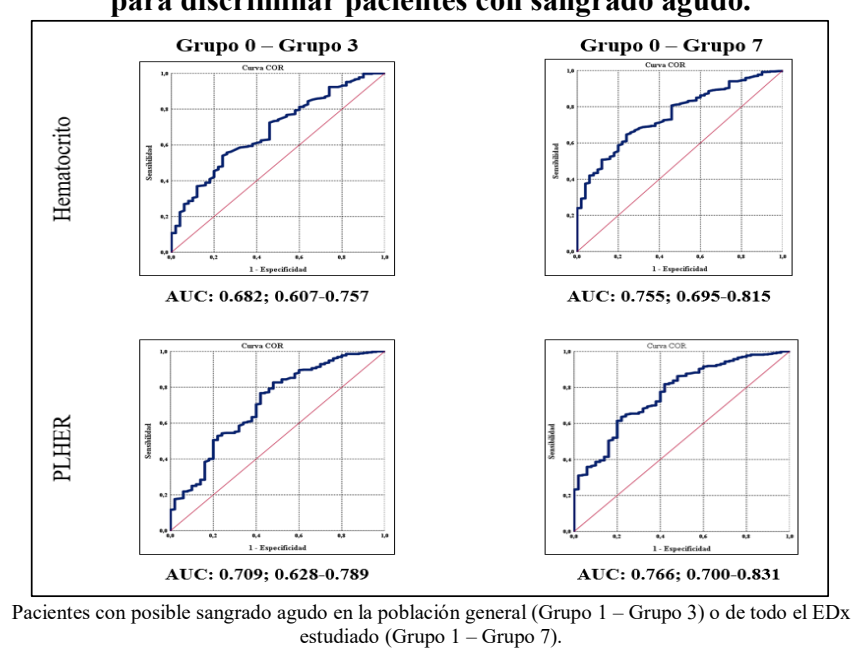

En la tabla 2 se presentan las capacidades operativas del PLHER y del hematocrito para discriminar pacientes con posible SA en la población general Grupo 1-3 o en todos los grupos del EDx estudiado Grupos 1-7.

Dado que previamente se estableció que la mediana del PLHER fue mayor en mujeres que en hombres, se calculó el AUC del PLHER y hematocrito, tanto en hombres como en mujeres, para discriminar pacientes con SA.

En mujeres las AUC del PLHER y hematocrito, respectivamente fueron 0.745 (IC95\%, 0.582-0.908) y 0.715 (IC95\%, 0.585-0.846), diferente a las estimaciones en hombres, donde las AUC del PLHER y hematocrito, respectivamente fueron 0.741 (IC95\%, 0.665-0.817) y 0.738 (IC95\%, 0.6720.804) (datos no mostrados).

Tabla 1: Capacidades operativas para discriminar pacientes con sangrado agudo.

\begin{tabular}{ccccc}
\hline \hline \multirow{2}{*}{$\begin{array}{c}\text { Capacidades } \\
\text { operativas }\end{array}$} & \begin{tabular}{c} 
Grupos \\
\cline { 2 - 5 }
\end{tabular} & $\begin{array}{c}\text { Grupos } \\
1-7\end{array}$ & $\begin{array}{c}\text { Grupos } \\
1-3\end{array}$ & $\begin{array}{c}\text { Grupos } \\
1-7\end{array}$ \\
\hline \hline $\begin{array}{c}\text { Punto de } \\
\text { corte }\end{array}$ & 5.413 & 6.337 & 40.2 & 40.2 \\
AUC & 0.709 & 0.766 & 0.682 & 0.755 \\
Sensibilidad & $76.7 \%$ & $64.0 \%$ & $54 \%$ & $64.6 \%$ \\
Especificidad & $58.0 \%$ & $78.0 \%$ & $76 \%$ & $76.0 \%$ \\
VPP & $92.8 \%$ & $97.2 \%$ & $94.1 \%$ & $97.0 \%$ \\
VPN & $26.1 \%$ & $15.2 \%$ & $19.0 \%$ & $15.1 \%$ \\
LR+ & 1.826 & 2.908 & 2.249 & 2.693 \\
LR- & 0.402 & 0.462 & 0.606 & 0.465 \\
\hline \hline
\end{tabular}

$\overline{\text { Se comparó el grupo control negativo con dos combinaciones de grupos de pacientes, Grupo } 1 \text { - Grupo }}$ 3 (pacientes con posible sangrado agudo) y todos los grupos de pacientes que conformaban el espectro diagnóstico (Grupo 1 - Grupo 7).

\section{DISCUSIÓN}

Esta investigación permitió comparar un parámetro hematológico utilizado como referente para monitorear los pacientes con SA, contra un constructo que surgió de los principios fisiológicos y distribución de poblaciones celulares que participan en la hemostasia.

La hemostasia es un proceso orientado a detener el sangrado en cualquier área corporal donde ocurra, sin interrumpir el flujo normal de sangre por un vaso sanguíneo (30). El SA altera principal y simultáneamente la distribución general de dos grupos celulares contenidos en la circulación general, los eritrocitos porque pasan en masa del espacio intra al extravascular y las plaquetas porque se movilizan hacia el sitio lesionado $(13,14)$.

Históricamente, el volumen de eritrocitos con respecto al total de la sangre se ha monitoreado con el hematocrito (13). Este parámetro expresa directamente cambios distributivos de los eritrocitos durante el SA, sin embargo, debido a que el 
método utilizado para calcularlo incluye la medición de capas de eritrocitos, el total celular y el plasma, en condiciones de perdidas plasmáticas masivas como la hipovolemia o hemorragia, la precisión para monitorear el sangrado disminuye por efectos de la hemoconcentración (14-17).

La utilidad del hematocrito como herramienta de evaluación de la gravedad inicial y monitoreo en la SE a pacientes con SA se ha cuestionado debido a la concepción teórica y fisiológica de la hemoconcentración esperada que acompaña a perdidas masivas de volumen $(15,17,18)$. Evidencia reciente ha identificado que, moderadamente (AUC: 0.72) la hemoglobina prehospitalaria logró predecir el choque hemorrágico en víctimas de trauma, y que la hemoglobina intrahospitalaria tenía mayor capacidad (AUC: 0.92) que el índice de choque de predecir esta manifestación clínica (19).

Cabe aclarar dos aspectos relevantes, las guías o estándares de monitoreo y manejo de pacientes con SA y choque hemorrágico recomiendan el uso del hematocrito como parámetro para conseguir metas y tomar decisiones clínicas, por otra parte, la conversión de hematocrito a hemoglobina al dividir por tres no es válida ni precisa, por lo tanto, los resultados de estudios sobre la hemoglobina no son extrapolables al hematocrito (20-22).

En general, los estudios que utilizaron la hemoglobina o el hematocrito para evaluación inicial y monitoreo del SA en la SE presentaron resultados contradictorios y variables en el AUC y otras capacidades operativas; asimismo, la mayoría se diseñaron para predecir mortalidad, estancia hospitalaria, entre otros desenlaces clínicos, y por último, orientan al clínico a mejorar la anamnesis, examen físico y aplicar nuevas herramientas diagnósticas no invasivas para identificar pacientes con SA o choque en fases tempranas $(20,21)$.

En nuestros resultados, el PLHER presentó mejor AUC y $\mathrm{LR}+$ que el hematocrito para discriminar pacientes con SA. El LR+ estimado para el PLHER indica al menos un incremento de $25 \%$ en la probabilidad para discriminar pacientes con SA comparado con el hematocrito.

El hallazgo más relevante de esta investigación se encontró en los grupos 1 y 2 clasificados con posible SA, donde el PLHER presentó diferencias estadísticas contra el control negativo. Los grupos 1 y 2 incluyeron pacientes con sospecha de SA, algunos con trauma ortopédico cerrado de huesos largos y pelvis, y otros con cualquier causa de sangrado. En contraste con el PLHER, el hematocrito estimado del control negativo no fue diferente al de los grupos 1 o 2 . Esto es un hallazgo relevante dado que con un punto de corte $\geq 6.037$ el PLHER podría discriminar pacientes con posible SA y asimismo puede ser una ventana de oportunidad para abrir los protocolos de aproximación al SA en las SE.

El siguiente paso es evaluar la consistencia de estos hallazgos en modelos animales controlando estrictamente el tiempo desde el inicio del sangrado y volumen de perdidas sanguíneas. Igualmente debe replicarse esta investigación en otros escenarios de atención de emergencias e incluyendo tamaños uniformes de muestras entre los grupos del espectro clínico.

Conflicto de Interés: Los autores declaran que NO hay conflicto de interés.

\section{REFERENCIAS}

1. Kumar R, Mills AM. Gastrointestinal bleeding. Emerg Med Clin North Am. 2011 May;29(2):239-52, viii. doi: 10.1016/j.emc.2011.01.003.

2. Whitaker L, Critchley HO. Abnormal uterine bleeding. Best Pract Res Clin Obstet Gynaecol. 2016 Jul;34:54-65. doi: 10.1016/j.bpobgyn.2015.11.012.

3. Özgönenel B, Zia A, Callaghan MU, Chitlur M, Rajpurkar M, Lusher JM. Emergency department visits in children with hemophilia. Pediatr Blood Cancer. 2013 Jul;60(7):1188-91. doi: 10.1002/pbc.24401.

4. Rubboli A, Becattini C, Verheugt FW. Incidence, clinical impact and risk of bleeding during oral anticoagulation therapy. World J Cardiol. 2011 Nov 26;3(11):351-8. doi: 10.4330/wjc.v3.i11.351

5. Lawton LD, Roncal S, Leonard E, Stack A, Dinh MM, Byrne CM, et al. The utility of Advanced Trauma Life Support (ATLS) clinical shock grading in assessment of trauma. Emerg Med J. 2014 May;31(5):384-9. doi: 10.1136/emermed-2012-201813.

6. Rose JS, Bair AE, Mandavia D, Kinser DJ. The UHP Ultrasound Protocol: A Novel Ultrasound Approach to the Empiric Evaluation of the Undifferentiated Hypotensive Patient. Am J Emerg Med. 2001;19:299-302.

7. Tomizawa M, Shinozaki F, Hasegawa R, Shirai Y, Motoyoshi Y, Sugiyama T, et al. Patient characteristics with high or low blood urea nitrogen in upper gastrointestinal bleeding. World J Gastroenterol. 2015 Jun 28;21(24):75005. doi: 10.3748/wjg.v21.i24.7500.

8. Ernst AA, Haynes ML, Nick TG, Weiss SJ. Usefulness of the blood urea nitrogen/creatinine ratio in gastrointestinal bleeding. Am J Emerg Med. 1999 Jan;17(1):70-2.

9. Stanley Adrian J, Laine Loren, Dalton Harry R, Ngu Jing H, Schultz Michael, Abazi Roseta et al. Comparison of risk scoring systems for patients presenting with upper gastrointestinal bleeding: international multicentre prospective study BMJ 2017; 356 :i6432

10. Institute of Medicine (US) Committee on Fluid Resuscitation for Combat Casualties; Pope A, French G, Longnecker DE, editors. Fluid Resuscitation: State of the Science for Treating Combat Casualties and Civilian Injuries. Washington (DC): National Academies Press (US); 1999. 2, Pathophysiology of Acute Hemorrhagic Shock. Available

from: https://www.ncbi.nlm.nih.gov/books/NBK224592/

11. Mei Z, Shi L, Wang B, Yang J, Xiao Z, Du P, et al. Prognostic role of pretreatment blood neutrophil-tolymphocyte ratio in advanced cancer survivors: A systematic review and meta-analysis of 66 cohort studies. Cancer Treat Rev. 2017 Jul;58:1-13. doi: 10.1016/j.ctrv.2017.05.005.

12. Karakonstantis S, Kalemaki D. Neutrophil to Lymphocyte Ratio As a Risk Stratification Tool for Older Adults with Pneumonia. J Am Geriatr Soc. 2017 Nov 20. doi: 10.1111/jgs.15206. 
13. Gale AJ. Continuing education course \#2: current understanding of hemostasis. Toxicol Pathol. 2011 Jan;39(1):273-80. doi: 10.1177/0192623310389474.

14. Mondal H, Budh DP. Hematocrit (HCT) [Updated 2019 Jun 3]. In: StatPearls [Internet]. Treasure Island (FL): StatPearls Publishing; 2019 Jan-. Available from: https://www.ncbi.nlm.nih.gov/books/NBK542276/

15. Taghavi S, Askari R. Hypovolemic Shock. [Updated 2019 Jun 18]. In: StatPearls [Internet]. Treasure Island (FL): StatPearls Publishing; 2019 Jan-. Available from: https://www.ncbi.nlm.nih.gov/books/NBK513297/

16. Balderas V, Bhore R, Lara LF, Spesivtseva J, Rockey DC. The hematocrit level in upper gastrointestinal hemorrhage: safety of endoscopy and outcomes. Am J Med. 2011 Oct;124(10):970-6. doi: 10.1016/j.amjmed.2011.04.032.

17. Valeri CR, Dennis RC, Ragno G, Macgregor H, Menzoian JO, Khuri SF. Limitations of the hematocrit level to assess the need for red blood cell transfusion in hypovolemic anemic patients. Transfusion. 2006 Mar;46(3):365-71.

18. Takanishi DM, Yu M, Lurie F, Biuk-Aghai E, Yamauchi H, Ho HC, et al. Peripheral blood hematocrit in critically ill surgical patients: an imprecise surrogate of true red blood cell volume. Anesth Analg. 2008 Jun;106(6):1808-12. doi: 10.1213/ane.0b013e3181731d7c.

19. Figueiredo S, Taconet C, Harrois A, Hamada S, Gauss T, Raux M, et al. How useful are hemoglobin concentration and its variations to predict significant hemorrhage in the early phase of trauma? A multicentric cohort study. Ann Intensive Care. 2018 Jul 6;8(1):76. doi: 10.1186/s13613-018-0420-8.

20. Cannon JW. Hemorrhagic Shock. N Engl J Med. 2018 Jan 25;378(4):370-379. doi: 10.1056/NEJMra1705649.

21. Quintó L, Aponte JJ, Menéndez C, Sacarlal J, Aide P, Espasa $\mathrm{M}$, et al. Relationship between haemoglobin and haematocrit in the definition of anaemia. Trop Med Int Health. 2006 Aug;11(8):1295-302.

22. Spahn DR, Bouillon B, Cerny V, Duranteau J, Filipescu D, Hunt BJ, et al. The European guideline on management of major bleeding and coagulopathy following trauma: fifth edition. Crit Care. 2019 Mar 27;23(1):98. doi: 10.1186/s13054-019-2347-3. 


\section{Material suplementario.}

Tabla 1s: Características generales.

\begin{tabular}{clrrrrr}
\hline \hline Variable & Espectro & Mínimo & Q1 & Mediana & Q3 & Máximo \\
\hline \hline PLHER & Grupo 0 & 3,026 & 4,363 & 5,089 & 6,274 & 9,405 \\
& Grupo 1 & 3,394 & 4,967 & 6,031 & 7,078 & 12,061 \\
& Grupo 2 & 4,222 & 5,487 & 6,349 & 7,754 & 18,811 \\
& Grupo 3 & 3,481 & 5,541 & 6,525 & 7,788 & 19,385 \\
& Grupo 4 & 4,681 & 6,368 & 7,648 & 8,805 & 17,029 \\
& Grupo 5 & 3,275 & 6,374 & 8,199 & 10,268 & 23,394 \\
& Grupo 6 & 3,341 & 6,774 & 9,315 & 15,040 & 29,153 \\
& Grupo 7 & 3,660 & 7,227 & 11,603 & 16,045 & 27,082 \\
& & & & & & \\
Hematocrito & Grupo 0 & 29,700 & 40,100 & 43,800 & 47,300 & 55,200 \\
& Grupo 1 & 33,000 & 42,300 & 44,300 & 45,800 & 49,500 \\
& Grupo 2 & 24,400 & 40,400 & 43,100 & 45,875 & 49,900 \\
& Grupo 3 & 22,600 & 32,800 & 38,100 & 42,400 & 52,000 \\
& Grupo 4 & 27,200 & 36,825 & 38,950 & 41,550 & 46,000 \\
& Grupo 5 & 21,300 & 25,900 & 30,200 & 36,300 & 56,900 \\
& Grupo 6 & 21,400 & 27,200 & 29,550 & 35,550 & 45,200 \\
& Grupo 7 & 21,40 & 23,30 & 25,80 & 34,20 & 55,40 \\
& & & & & & \\
Plaquetas & Grupo 0 & 150,00 & 194,75 & 224,00 & 264,25 & 395,00 \\
& Grupo 1 & 163,0 & 226,0 & 256,0 & 319,0 & 398,0 \\
& Grupo 2 & 158,0 & 239,5 & 276,5 & 328,0 & 621,0 \\
& Grupo 3 & 150,00 & 203,00 & 245,00 & 291,00 & 541,00 \\
& Grupo 4 & 186,0 & 253,0 & 299,0 & 347,8 & 579,0 \\
& Grupo 5 & 150,00 & 201,50 & 242,00 & 301,00 & 627,00 \\
& Grupo 6 & 151,0 & 221,0 & 307,5 & 419,5 & 723,0 \\
& Grupo 7 & 162,0 & 233,0 & 331,0 & 441,0 & 666,0 \\
\hline \hline & & & & & \\
& & & & & \\
\end{tabular}

\section{Abreviaturas.}

CDO: Clínica del Occidente S.A.

EDx: espectro diagnóstico

PLHER: radio plaqueta/hematocrito

SA: sangrado agudo

SE: sala de emergencias

SAM: sangrado agudo de causa médica

SAT: sangrado agudo de causa traumática 